\title{
Flatness and submersivity of discrete-time dynamical systems
}

\author{
Philippe Guillot $^{1}$ and Gilles Millérioux ${ }^{2}$
}

\begin{abstract}
This paper addresses flatness of discrete-time systems called difference flatness. A definition of flatness, that encompasses the standard ones, in particular backward and forward difference flatness, is introduced. It also allows to cope with systems which are not necessarily controllable or submersive. Besides, it considers nonlinear dynamical systems defined on general sets (without necessary special structures) which can be either continuous or discrete. Based on this definition, a result is established and stipulates that a flat and submersive nonlinear system is fully reachable (which is equivalent to fully controllable). Next, the special case of linear systems is considered leading to a necessary and sufficient condition.
\end{abstract}

Index Terms-flatness, nonlinear discrete-time systems

\section{INTRODUCTION}

This paper is concerned with flatness of discrete-time dynamical systems, which is called difference flatness. In the literature, difference flatness is presented as the discrete counterpart of differential flatness which was first introduced in [1] and was dedicated to continuous-time systems. For a discrete-time system, flatness means that the state variables as well as the inputs are written as a function of the so-called flat output, including forward and backward shifts in this output. Difference flatness has been addressed in a much lesser extent than differential flatness. It has been first reported in [2], [3] and it is motivated by the fact that some systems are intrinsically discrete (models of population growth, economy, biology, finance, discrete automata,...). Besides, it must be stressed that the property of flatness may not be preserved when a flat continuous-time system is discretized, even in the linear case (see [2] for a simple counter-example). Hence, flatness for sampled-data systems should preferably be addressed directly in discrete-time.

Various issues related to flatness have been investigated in the literature, like testing flatness for a specific output, searching flat outputs, constructing flat systems. Most issues are addressed for systems defined over sets with specific structures as the field of real numbers. Moreover, the properties of reversibility (also called invertibility, see [4]) of the systems and/or reachability and/or submersivity are most often assumed to hold. For example, for linear systems, a condition to characterize flatness and a method to construct

\footnotetext{
${ }^{1}$ Philippe Guillot is with the LAGA CNRS, Universite Paris 8, France philippe.guillot@univ-paris8.fr

${ }^{2}$ Gilles Millérioux is with the CRAN CNRS, Lorraine University, France gilles.millerioux@univ-lorraine.fr

This work was partly supported by the french PIA project "Lorraine Université d'Excellence", reference ANR-15-IDEX-04-LUE
}

a flat output are provided in [2]. The construction of a flat output is based on a change of variable involving the inverse of the reachability matrix. However, the method may not apply for non-reachable linear systems since the reachability matrix is singular in such a case. Still for linear systems, a computational approach for flat output test and construction is given in [5]. For the construction issue, it is proposed to transform the system into a canonical form (chain of integrators). Thus, the forward and backward difference flat outputs can be readily constructed by simple inspection. The set of flat outputs are canonical outputs (only one non-zero entry in the rows output matrix). The method, suited for MIMO linear systems, follows similar lines to the one proposed in [6] for SISO linear systems. However, reachability is assumed to hold. As for nonlinear systems, the paper [7] addresses the problem of dynamic feedback linearization. The paper [8] addresses the construction of flat outputs. The method requires the computation of so-called unimodular matrices. The papers [9], [10] introduce a method based on the transformations into a suitable normal form to construct flat outputs. But in all the aforementioned papers, the nonlinear system under consideration is assumed to have some specific properties: it is submersive, merophorphic or analytic.

The contribution of this paper is twofold. First, a definition of flatness, that encompasses the standard ones, in particular backward and forward difference flatness, is introduced. It also allows to cope with systems which are not necessarily controllable or submersive. Besides, it considers nonlinear dynamical systems defined on general sets (without necessary special structures) which can be either continuous or discrete. Then, based on this definition, a result is established and stipulates that a flat and submersive nonlinear system is fully reachable (which is equivalent to fully controllable). Next, the special case of linear systems is considered leading to a necessary and sufficient condition. The outline of the paper is the following. In Section II, a definition of difference flatness is given and its generality, compared to standard definitions, is discussed. Section III focuses on definitions of reachability and controllability which play a central role when addressing flatness. A distinction is made between local and global properties and the role of reversibility is discussed. Section IV is devoted to the main result which states that flatness and submersivity of nonlinear systems are sufficient conditions for full controllability. Next, the specific case of linear systems is considered. All along the paper, several examples which are deliberately simple illustrate the notions and the results. 


\section{DEFINITIONS AND PROBLEM STATEMENT}

Let us consider a discrete-time controlled dynamical system $\mathcal{S}$ described by

$$
\mathcal{S}: x_{k+1}=f\left(x_{k}, u_{k}\right)
$$

where $x_{k} \in \mathcal{X}$ is the internal state (the $i$-th component is denoted by $\left.x_{k}^{(i)}\right), u_{k} \in \mathcal{U}$ is the input, $f$ is the (one step) possibly nonlinear state transition function.

When the system defined by Equation (1) is linear, the sets $\mathcal{U}$ and $\mathcal{X}$ are finite dimensional vector spaces over the same field denoted by $\mathbb{F}$. In this case, the system is denoted by $\mathcal{L}$ and it admits the state space representation

$$
\mathcal{L}: x_{k+1}=A x_{k}+B u_{k}
$$

where $x_{k} \in \mathbb{F}^{n}, u_{k} \in \mathbb{F}^{m}$, with $n$ and $m$ being some nonzero natural integers. The matrices $A$ and $B$, of appropriate dimension, are respectively the dynamical matrix and the input matrix.

It is clear that any property which holds for the system $\mathcal{S}$ also applies for the system $\mathcal{L}$.

Let us consider the system $\mathcal{S}$ defined by Equation (1).

Definition 1 (flat output): Let $h$ be a possibly nonlinear function defined over the set $\mathcal{X} \times \mathcal{U}^{I}$ with $I$ a non negative integer. The function takes values in the set $\mathcal{Y}=\mathcal{U}$. For any integer $k \in \mathbb{N}$, let $y_{k}=h\left(x_{k}, u_{k+\ell_{0}}, \ldots, u_{k+\ell_{0}+I-1}\right)$ with $\ell_{0}$ an integer ranging in $\{-I+1, \cdots, 0\}$ (past and future inputs are possibly considered altogether). The function $h$ is said to be a flat output for the dynamical system $\mathcal{S}$ if there exists a non negative integer $k_{0}$ such that every variable of the system can be expressed as a function of $y_{k}$ and a finite number of its backward and/or forward iterates for $k \geq k_{0}$. In particular, there exist two functions $\mathcal{F}, \mathcal{G}$ and integers $r_{1}, r_{2}, s_{1}, s_{2}$ in $\mathbb{Z}$, satisfying $r_{1} \leq r_{2}$ and $s_{1} \leq s_{2}$, and such that, for $k \geq k_{0}$,

$$
\left\{\begin{array}{l}
x_{k}=\mathcal{F}\left(y_{k+r_{1}}, \cdots, y_{k+r_{2}}\right) \\
u_{k}=\mathcal{G}\left(y_{k+s_{1}}, \cdots, y_{k+s_{2}}\right)
\end{array}\right.
$$

Definition 2 (flat system): The dynamical system $\mathcal{S}$ is flat if it admits a flat output.

A flat output is a function but, hereafter and with a somehow abusive terminology, the value $y_{k}$ of the function $h$ will also be referred as flat output. If the flat output is exclusively a function of the state $x_{k}$, then the output and the system $\mathcal{S}$ are called 0-flat. If the flat output is a function of $x_{k}$ and only one (possibly shifted) input $u_{k+\ell_{0}}$, then the output and the system $\mathcal{S}$ are called 1-flat. More generally, if the flat output is a function of $I>0$ consecutive inputs, then the output and the system $\mathcal{S}$ are called $I$-flat. For linear systems like (2), a flat output must belong to $\mathbb{F}^{m}$ (square system). A 0flat output reads $y_{k}=C x_{k}$ where $C \in \mathbb{F}^{m \times n}$ while a $I$-flat output (for $I>0$ ) reads $y_{k}=C x_{k}+\sum_{\ell=\ell_{0}}^{\ell_{0}+I-1} D_{\ell} u_{k+\ell}$ where $D_{\ell} \in \mathbb{F}^{m \times m}$ (being $\ell$ a non negative integer). The notion of $I$-flat outputs has been discussed for example in [11], [12] for continuous-time systems or [5] for linear discrete-time systems (restricted to the case when only past or future inputs are considered in accordance with backward or forward flatness, that is $l_{0}=-I+1$ or $l_{0}=0$ ).

The following remarks are in order.

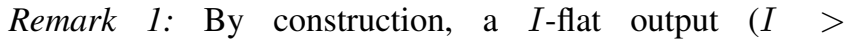
1) for the system described by (1) is a 1-flat output for the extended system defined by the extended state $\tilde{x}_{k}=\left[x_{k}, u_{k+\ell_{0}}, \cdots, u_{k+\ell_{0}+I-2}\right] \in \mathcal{X} \times \mathcal{U}^{I-1}$ and input $u_{k+\ell_{0}+I-1}$. Indeed, $y_{k}=h\left(x_{k}, u_{k+\ell_{0}}, \ldots, u_{k+\ell_{0}+I-1}\right)=$ $h\left(\tilde{x}_{k}, u_{k+\ell_{0}+I-1}\right)$ and it is clear that if $\tilde{x}_{k}$ can be expressed as a function of backward/forward shifts in the output, it also holds for $x_{k}$ and $u_{k}$ since $x_{k}$ and $u_{k}$ are involved in $\tilde{x}_{k}$.

Remark 2: It must be emphasized that flatness of the dynamical system $\mathcal{S}$ is a property characterizing only the state transition function $f$.

The following remarks point out the generality of Definition 1 .

Remark 3: Definition 1 involves relative integers $r_{i}$ and $s_{i}$ $(i=1,2)$ and generalizes the standard definitions of forward difference flatness when $r_{i} \geq 0$ and $s_{i} \geq 0(i=1,2)$ or backward difference flatness when $r_{i} \leq 0$ and $s_{i} \leq 0(i=$ 1,2 ) as discussed in [2] or [5] for Linear Time Invariant (LTI) systems. This generalization was first introduced in [13] but was restricted to fully controllable and invertible LTI discretetime systems. Besides, as already highlighted in [14] for the special case of switched linear systems, the consideration of relative integers $r_{i}$ and $s_{i}(i=1,2)$ in Definition 1 allows to consider SISO systems with arbitrary relative degree or MIMO systems with arbitrary inherent delay (in the sense of [15], [16]). The reader can refer to the example of Section IV for an illustration.

Remark 4: The integer $k_{0}$, introduced in Definition 1 , generalizes standard definitions to cope with special situations like non fully reachable systems. The example given in Subsection IV-C will illustrate such a purpose.

Remark 5: If $y_{k}$ is a flat output for (1), the $R$-shift output $y_{k}^{\prime}=y_{k+R}$ of (1), with $R$ a non negative integer, is still a flat output. Indeed, Equations (3) still hold by substituting $y_{k+i}$ by $y_{k+i-R}^{\prime}$. The integers $r_{i}$ and $s_{i}$ must be shifted accordingly. The output $y_{k}^{\prime}$ is well defined as a function $h$ involving the state $x_{k}$ and a finite number of consecutive inputs.

In this paper, we aim at studying the relationship between flatness obeying Definition 1 and the properties of controllability and reachability. A special emphasis, which can be considered as the main issue of the paper, is placed on the role of reversibility and submersivity.

Definition 3 (reversibility): The system $\mathcal{S}$ defined by Equation (1) is said to be reversible (also called invertible), if for any input $u \in \mathcal{U}$, the function $x \mapsto f(x, u)$ is invertible.

For the linear dynamical system $\mathcal{L}$ defined by (2), reversibility means that the dynamical matrix $A$ is invertible.

Definition 4 (submersivity): The dynamical system $\mathcal{S}$ is submersive if the state transition function $f$ is surjective (onto). In other words, the dynamical system $\mathcal{S}$ is submersive if for any state $x_{k}$ in $\mathcal{X}$, there exists a control $u_{k-1}$ in $\mathcal{U}$ and a one-step preimage $x_{k-1}$ of $x_{k}$ such that $x_{k-1}$ reaches $x_{k}$ by applying the control $u_{k-1}$.

When we concentrate on the linear system $\mathcal{L}$ described by Equation (2), the following remarks can be made.

Remark 6: As a linear function is surjective if and only if it is full rank, it holds that the system $\mathcal{L}$ is submersive if and only if

$$
\operatorname{rank}[A B]=n
$$


As a consequence, the following remark holds:

Remark 7: If $A$ is invertible then $\operatorname{rank}[A B]=n$ and thus, the dynamical system $\mathcal{L}$ is submersive.

In other words, reversible systems belong to the special class of submersive systems. In the sequel, we discuss the role of reversibility and submersivity when addressing controllability and reachability. Indeed, as shown later, they play a central role to characterize flatness.

\section{REACHABILITY AND CONTROLLABILITY}

It is recalled that controllability and reachability of a system refer to the ability to steer the state, via the control input, to some locations in the state space. Many definitions and propositions given in this section can be found in standard references like [17], [18], [19], [20]. However, let us notice that, in the literature, they are mostly presented for systems defined over $\mathbb{R}$. Here, the sets over which the system described by (1) is defined is not assumed to have a special structure. Besides, the subtleties induced by the reversibility or the submersivity of (1) which may hold or not are deeply highlighted.

1) The general case: In the sequel, $x^{i}$ and $x^{f}$ will denote respectively an initial and final (or target) state in $\mathcal{X}$.

Definition 5 (reachability from $x^{i}$ of a state): A state $x^{f}$ of $\mathcal{S}$ is reachable from $x^{i}$ if there exists an integer $N>0$ and a non empty input sequence $\left(u_{0}, \cdots, u_{N-1}\right)$ of length $N$ that steers $x^{i}$ to $x^{f}$.

Definition 6 (reachability from $x^{i}$ of a system): The dynamical system $\mathcal{S}$ is reachable from $x^{i}$ if any state $x^{f}$ is reachable from $x^{i}$.

Definition 7 (full reachability of a system): The dynamical system $\mathcal{S}$ is fully reachable if, for any initial state $x^{i}$, it is reachable from $x^{i}$.

The reachability from $x^{i}$ of a system is also called local reachability or forward accessibility (see [21] and references therein).

The following proposition highlights a connection between reachability and submersivity.

Proposition 1: If there exists an internal state $x^{i}$ such that the dynamical system $\mathcal{S}$ is reachable from $x^{i}$ then $\mathcal{S}$ is submersive.

Indeed, if there exists an internal state $x^{i}$ such that the dynamical system $\mathcal{S}$ is reachable from $x^{i}$ then there exists a non empty control sequence that steers $x^{i}$ to any target state $x^{f}$. The last step just before reaching $x^{f}$ provides the state and the control of which existence proves the submersivity. This result was already established for nonlinear systems defined over the field of real numbers in [22]. It will be shown further that for a linear dynamical system $\mathcal{L}$ described by Equation (2), Definition 6 and Definition 7 are equivalent.

On the other hand, reachability from a given initial state $x^{i}$ and full reachability are, in general, not equivalent for nonlinear dynamical systems, as shown by the following counter-example. Let us consider the system $\mathcal{S}$ described by Equation (1) and defined on the finite set $\mathcal{X}=\left\{x^{0}, x^{1}\right\}$. The input belongs to the finite alphabet $\{0,1\}$ and the state transition function $f$ verifies:

$$
f\left(x^{0}, 0\right)=x^{0}, f\left(x^{0}, 1\right)=x^{1}, f\left(x^{1}, 0\right)=f\left(x^{1}, 1\right)=x^{1}
$$

This system is not fully reachable since, clearly, it is reachable from $x^{0}$ but not from $x^{1}$.

Definition 8 (controllability to $x^{f}$ of a state): A state $x^{i}$ of the dynamical system $\mathcal{S}$ is controllable to $x^{f}$ if there exists an integer $N>0$ and a non empty input sequence $\left(u_{0}, \cdots, u_{N-1}\right)$ of length $N$ that steers $x^{i}$ to $x^{f}$.

Definition 9 (controllability to $x^{f}$ of a system): The dynamical system $\mathcal{S}$ is controllable to $x^{f}$ if any state $x^{i}$ is controllable to $x^{f}$.

Definition 10 (full controllability of a system): The dynamical system $\mathcal{S}$ is fully controllable if, for any target state $x^{f}$, it is controllable to $x^{f}$.

The controllability to $x^{f}$ of a system is also called local controllability or backward accessibility (see [21] and references therein).

It will be shown further that, unlike reachability, even for a linear dynamical system $\mathcal{L}$ described by Equation (2), Definition 9 and Definition 10 are not equivalent.

Remark 8: It can be noticed that a state $x^{f}$ is reachable from $x^{i}$ is equivalent to that the state $x^{i}$ is controllable to $x^{f}$. Similarly, a system is fully controllable if and only if it is fully reachable.

2) The special case of linear systems: For the linear system $\mathcal{L}$ described by Equation (2), reachability and controllability properties can be expressed in terms of rank matrix conditions. They are based on the relation directly obtained by iterating $n$ times Equation (2) from an initial condition $x^{i} \in \mathbb{F}^{n}$ at time $k=0$ and reaching the state $x^{f} \in \mathbb{F}^{n}$ at time $k=n$

$$
x^{f}=A^{n} x^{i}+Q_{c} u_{0}^{n}
$$

where $u_{0}^{n}$ denotes the vector $\left[\begin{array}{llll}u_{0}^{T} & \cdots & u_{n-1}^{T}\end{array}\right]^{T}\left(\begin{array}{c}T \\ \end{array}\right.$ stands for the transposition) and where $Q_{c}$ is defined as

$$
Q_{c}=\left[\begin{array}{llll}
B & A B & \cdots & A^{n-1} B
\end{array}\right]
$$

The vector $u_{0}^{n}$ can be equivalently considered as an input sequence of finite length $n$. The matrix $Q_{c}$ is called the reachability matrix.

Let $x^{i}$ be a given initial state. From equality (5), any target state $x^{f}$ can be reached from $x^{i}$ in at most $n$ steps if and only if there exists an input sequence $u_{0}^{n}$ of length $n$ such that $Q_{c} u_{0}^{n}=x^{f}-A^{n} x^{i}$ which is equivalent to that

$$
\forall x^{f} \in \mathbb{F}^{n}, x^{f}-A^{n} x^{i} \in \mathcal{I} \mathrm{m}\left(Q_{c}\right)
$$

where $\mathcal{I} \mathrm{m}$ denotes the range space.

It can be noticed that if there exists a state $x^{i}$ such that the dynamical system $\mathcal{L}$ is reachable from $x^{i}$, then, as the above relation holds for any $x^{f}$, it is also satisfied for any other $x^{i}$. Thus, as opposed to nonlinear systems, the reachability of the linear system $\mathcal{L}$ defined by Equation (2) from a given initial state $x^{i}$ implies its full reachability. From Remark 8, this is equivalent to the full controllability of $\mathcal{L}$. Hence, the following proposition holds.

Proposition 2: The following properties are equivalent: a) There exists an initial state $x^{i} \in \mathbb{F}^{n}$ such that $\mathcal{L}$ is reachable from $x^{i}$

b) $\mathcal{L}$ is fully reachable

c) $\mathcal{L}$ is fully controllable 
From (6), one obtains the well-established Kalman reachability rank condition which stipulates that the linear dynamical system $\mathcal{L}$ described by Equation (2) is fully reachable (which is equivalent to fully controllable) if and only if

$$
\operatorname{rank} Q_{c}=n
$$

On the other hand, the controllability to state $x^{f}$ means that for any initial state $x^{i} \in \mathbb{F}^{n}$, there exists an input sequence $u_{0}^{n}$ of length $n$ such that $Q_{c} u_{0}^{n}=x^{f}-A^{n} x^{i}$. Hence, one has the following proposition:

Proposition 3: The linear dynamical system $\mathcal{L}$ described by Equation (2) is controllable to $x^{f}$ if and only if

$$
\forall x^{i} \in \mathbb{F}^{n}, \quad x^{f}-A^{n} x^{i} \in \mathcal{I} \mathrm{m}\left(Q_{c}\right)
$$

Thus, unlike reachability, a linear dynamical system may be controllable to a given state $x^{f}$ without being fully controllable. It holds in particular for non reversible systems as illustrated by the following example. Let us consider the 2dimensional linear system described by Equation (2) with state space matrices

$$
A=\left[\begin{array}{ll}
a & 0 \\
0 & 0
\end{array}\right], \quad B=\left[\begin{array}{l}
1 \\
0
\end{array}\right]
$$

The reachability matrix is $Q_{c}=\left[\begin{array}{ll}1 & a \\ 0 & 0\end{array}\right]$ whose rank is 1 . Thus, the system is not fully controllable. On the other hand, it is controllable to state $\left[\begin{array}{ll}1 & 0\end{array}\right]^{T}$ but not to state $\left[\begin{array}{ll}0 & 1\end{array}\right]^{T}$. This is explained by the fact that in this special case, the matrix $A$ is singular, that is the linear system is not reversible. On the other hand, if $A$ is full rank, the following proposition holds.

Proposition 4: If the linear dynamical system $\mathcal{L}$ described by Equation (2) is reversible, that is $A$ is non-singular, the following properties are equivalent.

a) There exists an initial state $x^{i} \in \mathbb{F}^{n}$ such that $\mathcal{L}$ is reachable from $x^{i}$

b) $\mathcal{L}$ is fully reachable

c) $\mathcal{L}$ is fully controllable

d) There exists a target state $x^{f} \in \mathbb{F}^{n}$ such that $\mathcal{L}$ is controllable to $x^{f}$

Let us further consider the reachability from zero and the controllability to zero. From (6) and setting $x^{i}=0$, the following proposition applies regardless whether the linear dynamical system (2) is reversible or not.

Proposition 5: The linear dynamical system $\mathcal{L}$ described by Equation (2) is reachable from zero if and only if

$$
\operatorname{rank} Q_{c}=n
$$

On the other hand, the linear dynamical system $\mathcal{L}$ is controllable to zero if and only if, for any initial state $x^{i}$, there exists an input sequence $u_{0}^{n}$ of length $n$ such that $Q_{c} u_{0}^{n}=-A^{n} x^{i}$. Thus, the following proposition applies

Proposition 6: The linear dynamical system $\mathcal{L}$ described by Equation (2) is controllable to zero if and only if

$$
\mathcal{I m} A^{n} \subseteq \mathcal{I m} Q_{c}
$$

The following remark is straightforward for the linear dynamical system $\mathcal{L}$.
Remark 9: Reachability from zero of the linear system $\mathcal{L}$ described by Equation (2) implies controllability to zero of $\mathcal{L}$. If $A$ is nilpotent then the linear dynamical system $\mathcal{L}$ is controllable to zero.

\section{MAIN RESULT}

In this section, a relation between flatness and full controllability (which is equivalent to full reachability according to Remark 8) is presented. It is shown that submersivity is a specific property that must be considered in this regard.

Motivated by Remark 5, if Equations (3) hold, we can assume without any restriction that the dynamical system $\mathcal{S}$ admits a flat output such that

$$
\left\{\begin{array}{l}
x_{k}=\mathcal{F}\left(y_{k-r+1}, \cdots, y_{k}\right) \\
u_{k}=\mathcal{G}\left(y_{k-s+1}, \cdots, y_{k}\right)
\end{array}\right.
$$

where $r$ and $s$ are appropriate positive integers.

Before giving the main theorem, next subsection addresses the property of left-invertibility which, in addition to controllability and reachability, also plays a central role when addressing flatness.

\section{A. Left invertibility}

There exist different definitions of left invertibility. We recall the definition used here, which is in accordance with the ones proposed in [15], [16], [23] in a linear context and in [24] in a nonlinear context.

Definition 11 (Left invertibility): The dynamical system $\mathcal{S}$ is said to be left invertible if, for a given (infinite length) output sequence $(y)$ produced by $\mathcal{S}$, the (infinite length) input sequence $(u)$ that produces $(y)$ is unique.

In other words, the function (not given explicitly here) that defines the output sequence $(y)$ from the input sequence $(u)$ is injective. If the system $\mathcal{S}$ is left invertible, then there exists a system that produces the input from the output. By definition, such a system is called a left inverse of $\mathcal{S}$ and is denoted by $\mathcal{S}^{-1}$.

Remark 10: The condition in Definition 11 only involves output sequences $(y)$ that are effectively produced by the system $\mathcal{S}$. There is no restriction on other sequences that are not an output of $\mathcal{S}$. Thus, a given dynamical system may admit several left inverses which differ from each other by input sequences that are not produced by $\mathcal{S}$.

The equality involving the function $\mathcal{G}$ in Equations (10) means that the input of a flat system can be recovered from the output sequence. It acts as the input/output equation of a flat system. Hence, left invertibility is a necessary condition for flatness and a left inverse, called canonical left inverse, can be defined from $\mathcal{G}$ as follows.

Definition 12 (Canonical left inverse of a flat system): Let us assume that the dynamical system $\mathcal{S}$ is flat with flat output $y_{k}=h\left(x_{k}\right)$ (0-flat) or $y_{k}=h\left(x_{k}, u_{k}\right)$ (1-flat). Its canonical left inverse $\mathcal{S}^{-1}$ is defined by:

1) The internal state $(y)$ in the set of $s$-dimensional vectors of components in $\mathcal{Y}$;

2) The dynamic equation as a shift fed by the inputs $y_{k-i} \in$ $\mathcal{Y}$ 
3) The output function as the function $\mathcal{G}$ involved in Equation (10).

Remark 11: Note that the internal state of system $\mathcal{S}$ is not involved in the definition of the canonical left inverse. But if needed, it may be recovered from the internal state of $\mathcal{S}^{-1}$ by the function $\mathcal{F}$ involved in Equation (10).

Remark 12: For $I$-flat outputs with $I>1$, the canonical left inverse can be defined from the extended system as explained in Remark 1 and considering the $I$-flat output of $\mathcal{S}$ as a 1-flat output for the extended system of $\mathcal{S}$.

Remark 13: For left-invertible linear systems described by (2) and defined over the field of real-numbers (the generalization to any field is possible), the flat outputs can be characterized in terms of invariant zeros. Indeed, it has been shown in [13] or in [14] (considering that a linear system is a special switched linear one) that a linear system like (2), assumed to be left-invertible, is flat according to Definition 1, if and only if the dynamics of the left-inverse is trivial, a terminology introduced in [4]. It is equivalent to stipulate that either, there are no invariant zeros (see [25] for a well-admitted definition of zeros in MIMO systems). In this case, the system is strongly observable [26] or they are all equal to zero. It is also in accordance with the result given in [5] regarding forward difference flatness.

\section{B. Main theorem}

We are now able to state the main result of the paper.

Theorem 1: If the dynamical system $\mathcal{S}$ is flat and submersive, then it is fully controllable (which is equivalent to fully reachable according to Remark 8).

Proof 1: Let us assume that $\mathcal{S}$ is flat with flat output $y_{k}=h\left(x_{k}\right)$ (0-flat) or $y_{k}=h\left(x_{k}, u_{k}\right)$ (1-flat) obeying Equations (10) and that $\mathcal{S}$ is submersive. Let $x$ and $z$ be two arbitrary internal states in $\mathcal{X}$. It must be shown that there exists a finite input sequence that steers the state $x$ to the state $z$.

As the system $\mathcal{S}$ is supposed to be submersive, there exists an initial state $x^{0}$ and an input sequence $\left(u_{z}\right)$ of finite length $r$ that steers the state $x^{0}$ to the state $z$. Let us denote by $\left(y_{z}\right)$ the corresponding output sequence of length $r$. Since $\mathcal{S}$ is flat, one has $z=\mathcal{F}\left(\left(y_{z}\right)\right)$. Similarly, there exists an initial state $x^{\prime}$ and an input sequence $\left(u_{x}\right)$ of finite length $r$ that steers the state $x^{\prime}$ to the state $x$. Let us denote by $\left(y_{x}\right)$ the corresponding output sequence of length $r$. Since $\mathcal{S}$ is flat, one has $x=\mathcal{F}\left(\left(y_{x}\right)\right)$.

In order to show that there exists an input sequence denoted by $(u)$, that steers system $\mathcal{S}$ from $x$ to $z$ and that produces the output sequence $\left(y_{z}\right)$, let us consider the canonical left inverse $\mathcal{S}^{-1}$ of $\mathcal{S}$, initialized to state $\left(y_{x}\right)$ and forced by the sequence $\left(y_{z}\right)$. It turns out that, by definition of the left-inverse, the state reached by $\mathcal{S}^{-1}$ is $\left(y_{z}\right)$ and the corresponding $r$-length output sequence is the expected sequence $(u)$. Indeed, when $(u)$ forces system $\mathcal{S}$ initialized to $x$, the corresponding output is the sequence $\left(y_{z}\right)$ and since $z=\mathcal{F}\left(\left(y_{z}\right)\right)$, it means that the final state reached after $r$ steps is precisely $z$. Finally, if the dynamical system $\mathcal{S}$ is $I$-flat with $I>1$, the same reasoning applies when considering, as stressed in Remark 1, the extended system of $\mathcal{S}$ with extended state $\tilde{x}_{k}$, the extended canonical inverse as defined in Remark 12 and pointing out that submersivity and full reachability of the extended system of $\mathcal{S}$ imply, by construction, submersivity and full reachability of $S$.

The result applies for dynamical systems defined on general sets without necessary particular structures. The sets can be either continuous or discrete. Regarding possible singularities, they may be disregarded by defining sets where the singular points are removed. For sets where a measure can be defined, the result must be understood as generic (it holds everywhere except on sets of measure zero). Finally, if the sets are considered around particular points, the result must be considered as local.

The following example simply illustrates Theorem 1. Let us consider the nonlinear system defined over $\mathbb{R}_{*}=\mathbb{R} \backslash\{0\}$ (the singular value 0 is thereby omitted) where $\mathbb{R}$ denotes the field of real numbers. The state transition function $\left(x_{k}, u_{k}\right) \in$ $\mathbb{R}_{*}^{2} \times \mathbb{R}_{*} \mapsto x_{k+1}=f\left(x_{k}, u_{k}\right) \in \mathbb{R}_{*}^{2}$ is defined as

$$
\left\{\begin{array}{l}
x_{k+1}^{(1)}=x_{k}^{(2)} \cdot u_{k} \\
x_{k+1}^{(2)}=u_{k}
\end{array}\right.
$$

Such a system is flat since $y_{k}=x_{k}^{(2)}$ is a flat output (0-flat). Indeed, for $k \geq 0, x_{k}^{(2)}=y_{k}, x_{k}^{(1)}=y_{k-1} \cdot y_{k}\left(r_{1}=-1\right.$ and $r_{2}=0$ in Equation (3)). Furthermore, it holds that $u_{k}=y_{k+1}$ $\left(s_{1}=s_{2}=1\right.$ in Equation (3)) and this equation defines the canonical left inverse of (11). It is also submersive since, for any pair $\left(\bar{x}^{1}, \bar{x}^{2}\right) \in \mathbb{R}_{*}^{2} \times \mathbb{R}_{*}^{2}$, there exists a control $u_{0}=\bar{x}^{2} \in$ $\mathbb{R}_{*}$ and a preimage $\left(x^{1}, x^{2}=\bar{x}^{1} / \bar{x}^{2}\right) \in \mathbb{R}_{*}^{2} \times \mathbb{R}_{*}^{2}$ such that $f\left(x^{1}, x^{2}\right)=\left(\bar{x}^{2}, \bar{x}^{2}\right)$. Finally, the system is fully controllable on $\mathbb{R}_{*}^{2}$. Indeed, for any target state $\left(\bar{x}^{1}, \bar{x}^{2}\right) \in \mathbb{R}_{*}^{2} \times \mathbb{R}_{*}^{2}$ and any initial state in $\left(x^{1}, x^{2}\right) \in \mathbb{R}_{*}^{2} \times \mathbb{R}_{*}^{2}$, there exists a control sequence of length equal to 2 that steers $\left(x^{1}, x^{2}\right)$ to $\left(\bar{x}^{1}, \bar{x}^{2}\right)$. The control verifies $u_{0}=\bar{x}^{1} / \bar{x}^{2}$ and $u_{1}=\bar{x}^{2}$. Let us note that the output $y_{k}=u_{k}$ is another flat output (1-flat). It holds that $x_{k}^{(2)}=y_{k-1}, x_{k}^{(1)}=y_{k-2} \cdot y_{k-1}\left(r_{1}=-2\right.$ and $r_{2}=-1$ in Equation (3)) and $u_{k}=y_{k}\left(s_{1}=s_{2}=0\right.$ in Equation (3)). That illustrates Remark 3 which points out that the integers $r_{i}$ and $s_{i}$ depend on the relative degree for a SISO system. Here, the relative degree of (11) with respect to $y_{k}=x_{k}^{(2)}$ is 1 whereas it is 0 with respect to $y_{k}=u_{k}$.

\section{The linear case}

In the linear case, unlike nonlinear systems, the following theorem (see for example [2]) holds.

Theorem 2: If the linear dynamical system $\mathcal{L}$ is fully controllable (which is equivalent to fully reachable) then it is flat.

Proof 2: The proof is given in [2] and essentially shows that if the system $\mathcal{L}$ is fully controllable (which is equivalent to fully reachable), then, there exists a linear change of coordinates involving the reachability matrix $Q_{c}$, such that in the new coordinate space, both the state and the input can be expressed in terms of forward output shifts. The flat output is given by the linear combination of the state obtained from the last row of the inverse reachability matrix $Q_{c}$, up to a constant factor. Hence, the system is 0 -flat. 
Corollary 1: The linear dynamical system $\mathcal{L}$ is flat and submersive if and only if it is fully reachable (which is equivalent to fully controllable).

Proof 3: Consider the linear dynamical system $\mathcal{L}$. According to Theorem 2, if it is fully controllable then it is flat. Moreover, according to Proposition 1, it is also submersive, which proves the necessity. The sufficiency is straightforward by considering Theorem 1.

As a consequence of this corollary, a linear flat system with flat output $y_{k}$ does not necessarily implies that the system is fully reachable. However, the following theorem stipulates that for linear systems, controllability to the origin is a necessary condition for flatness.

Theorem 3: If the linear system $\mathcal{L}$ is flat, then it is controllable to the origin.

Proof 4: According to Remark 13, the linear system $\mathcal{L}$ is flat if and only if either it admits no invariant zeros or they are all equal to zero. Hence, if the system is not controllable, the uncontrollable subspace is spanned by the eigenvectors of the zero eigenvalues and therefore is controllable to the origin.

As a consequence, the uncontrollable part of a flat system is stable and thus, a flat system is necessarily stabilizable.

The following example illustrates Corollary 1 and Theorem 3 . Consider a linear system $\mathcal{L}$ with

$$
A=\left[\begin{array}{cc}
6 & 8 \\
-3 & -4
\end{array}\right], \quad B=\left[\begin{array}{c}
2 \\
-1
\end{array}\right] .
$$

The system is flat since $y_{k}=\left[\begin{array}{ll}2 & 3\end{array}\right] x_{k}$ and $y_{k}=\left[\begin{array}{ll}6 & 8\end{array}\right] x_{k}+$ $2 u_{k}+u_{k+1}$ are respectively 0 -flat and 2 -flat outputs. Indeed, the equalities (3) of Definition 1 apply for $k \geq k_{0}=1$ (the initial condition cannot be recovered) and read respectively

$$
\left\{\begin{array}{l}
x_{k}^{(1)}=2 y_{k} \\
x_{k}^{(2)}=-y_{k} \\
u_{k}=y_{k+1}-2 y_{k}
\end{array}, \quad\left\{\begin{aligned}
x_{k}^{(1)} & =2 y_{k-2} \\
x_{k}^{(2)} & =-y_{k-2} \\
u_{k} & =y_{k-1}-2 y_{k-2}
\end{aligned}\right.\right.
$$

The reachability matrix reads

$$
Q_{c}=\left[\begin{array}{cc}
2 & 4 \\
-1 & -2
\end{array}\right]
$$

. Since $\operatorname{rank} Q_{c}=1$, thus the system is not fully reachable. Furthermore, $\operatorname{rank}[A B]=1$ thus the system is not submersive. That explains why the system is flat whereas it is not fully controllable. On the other hand, $\operatorname{Im} A^{2}$ is spanned by $\left[\begin{array}{ll}2 & -1\end{array}\right]^{T}$ and $\operatorname{Im} Q_{c}$ is also spanned by $[2-1]^{T}$. Thus, (9) is fulfilled and the system is controllable to zero.

\section{CONCLUSION}

This paper has investigated the property of flatness of discrete-time systems. A definition of flatness that encompasses the standard ones, in particular backward and forward difference flatness, has been introduced. It also allows to cope with systems which are not necessarily controllable or submersive. Besides, it considers nonlinear dynamical systems defined on general sets (without necessary special structures) which can be either continuous or discrete. Then, based on this definition, it has been established that a flat and submersive nonlinear system is fully reachable (which is equivalene to fully controllable). It has been shown that the equivalence holds for the special case of linear systems.

\section{REFERENCES}

[1] M. Fliess, J. Levine, P. Martin, and P. Rouchon. Flatness and defect of non-linear systems: introductory theory and examples. Int. Jour. of Control, 61(6):1327-1361, 1995.

[2] H. Sira-Ramirez and S. K. Agrawal. Differentially Flat Systems. Marcel Dekker, New York, 2004.

[3] M. Fliess and R. Marquez. Toward a module theoretic approach to discrete-time linear predictive control. International Journal of Control, 73:606-623, 2000.

[4] M. Fliess. Reversible linear and nonlinear discrete-time dynamics. IEEE Trans. Automat. Contr., 37:1144-1153, 1992.

[5] S. Z. Yong, B. Paden, and E. Frazzoli. Computational methods for MIMO flat linear systems: Flat output characterization, test and tracking control. In Proc. of the American Control Conference (ACC'2015), Chicago, Il, USA, July 1-3 2015.

[6] G. Millérioux. A constructive approach for the design of finite time self-synchronizing coupled systems with unknown inputs. In Proc. of the International Symposium on Nonlinear Theory and its Applications (NOLTA 2010), Krakow, Poland, September 2010.

[7] A. Kaldmae and Ü Kotta. On flatness of discrete-time nonlinear systems. In Proc. of the 9th IFAC Symposium on Nonlinear Control Systems, Toulouse, France, 2013.

[8] K. Sato. On an algorithm for checking whether or not a nonlinear discretetime system is difference flat. In Proc. of 20th International Symposium on Mathematical Theory of Networks and Systems, Melbourne, Australia, July 2012.

[9] B. Kolar, A. Kaldmäe, M. Schöberl, Ü. Kotta, and K. Schlacher. Construction of flat outputs of nonlinear discrete-time systems in a geometric and an algebraic framework. In NOLCOS 2016, 2016.

[10] B. Kolar, M. Schöberl, and K. Schlacher. A decompsition procedure for the construction of flat outputs of discrete-time nonlinear control systems. In 22nd International Symposium on Mathematical Theory of Networks and Systems, 2016.

[11] G. Rigatos. Nonlinear Control and Filtering Using Differential Flatness Approaches. Applications to Electromechanical Systems, chapter Differential Flatness Theory and Flatness-Based Control. Studies in Systems, Decision and Control. Springer, 2015.

[12] S. P. Pereira da Silva. Flatness of nonlinear control systems : a cartankähler approach. Proc. Mathematical Theory of Networks et Systems MTNS'2000, 012000.

[13] J. Daafouz, M. Fliess, and G. Millérioux. Une approche intrinsèque des observateurs linéaires à entrées inconnues. In Proc. of the Conférence Internationale Francophone d'Automatique, Bordeaux, May 2006.

[14] G. Millérioux and J. Daafouz. Flatness of switched linear discrete-time systems. IEEE Transactions on Automatic Control, 54(3):615-619, March 2009.

[15] M. K. Sain and J. L. Massey. Invertibility of linear time-invariant dynamical systems. IEEE Transactions on Automatic Control, 14:141149, 1969.

[16] L. M. Silverman. Inversion of multivariable linear systems. IEEE Trans. Automatic Control, 14(3):270-276, June 1969.

[17] T. Fortmann and K. Hitz. An introduction to linear control systems. Marcel Dekker, Inc, 1977.

[18] D. E. Kirk. optimal control theory: an introduction. Printice-Hall, Inc, 1970.

[19] B. D. O Andersen and J. B. Moore. Optimal filtering. Dover Publications, Inc, 1979.

[20] A. R. Liu. Stochastic observability, reconstructibility, controllability, and reachability. $\mathrm{PhD}$ thesis, UC San Diego, 2011

[21] Y. Kawano and T. Ohtsuka. Commutative algebraic methods for controllability of discrete-time polynomial systems. International Journal of Control, 13(2):343-351, 2016.

[22] J. W Grizzle. A linear algebraic framework for the analysis of discretetime nonlinear systems. SIAM Journal on Control and Optimization, 31(4):1026-1044, 1993.

[23] T. Floquet and JP. Barbot. State and unknown input estimation for linear discrete-time systems. Automatica, 42(11):1883-1889, 2006.

[24] S. N. Singh. A modified algorithm for invertibility in nonlinear systems. IEEE Trans. Automat. Control, 26:595-598, 1981.

[25] H. H. Rosenbrock. State Space and Multivariable Theory. London: Nelson, 1970.

[26] M. L. J. Hautus. Strong detectability and observers. Systems and Control letters, 50:353-368, 1983. 